\title{
3D Printing Technology and its Development Trend
}

\author{
Yingying Zhao ${ }^{1, a}$, Xueren Dong ${ }^{1, b}$ and Xiuxia Zhao ${ }^{1, \mathrm{c}}$ \\ ${ }^{1}$ School of Mechanical Engineering, University of Jinan,Shandong 250000,China \\ a Zhaoyingyingwc@163.com, bost_dongxr@ujn.edu.cn, 'me_zhaoqx@ujn.edu.cn
}

Keywords: 3D printing technology, application, development trend

Abstract. 3D printing is an advanced manufacturing technology which is developing rapidly in near thirty years. Its advantages lie in simplifying producing process, reducing material waste, lowering production costs and meeting the spacial needs of different customers. At present, 3D printing is widely applied in various fields, such as mold manufacturing industry, aerospace, medial treatment etc. In this paper, the working principle and classification of 3D printing technology are elaborated in detail, the exiting problems are analyzed and the application trends of 3D technology are prospected in further.

\section{Introduction}

3D printing, also known as Additive Manufacturing, belongs to Rapid Prototyping Manufacturing. Different from the traditional manufacturing processes, which use milling, boring, plane and many other machining processes to remove redundant parts of raw materials or blanks to get the final parts or products, 3D printing technology is a method that makes three-dimensional solid objects from a computerized model. It establishes digital model by three-dimensional design software, then partitioning the model into layer-by-layer cross sections. Afterwards, generating date information that can be identified by 3D printers. Then, According to the information, 3D printers will use adhesive materials to print the sections step by step and bond these sections together. Until entities are generated.

\section{The classification of $3 \mathrm{D}$ printing technology}

Fused Deposition Modeling (FDM). Heating and melting the filamentous materials in the spray head. Afterwards, in accordance with the relevant provisions, the molten materials are extruded onto the worktable or the previous layer of the cured materials by the nozzle. The materials start to cure when the temperature reaches the curing temperature and form cross section of the workpiece. Then adjust the height of the nozzle, and applying the next layer. Repeating the process until eventually forming three-dimensional workpiece[1].

Laminated Object Manufacturing(LOM). Coating hot melt adhesive on the surface of materials, then bonding with the previous layer after being rolled by hot-press roller. Using the laser beam to cut the current paper according to the slice data. Repeating the process until the parts formed[2].

Selective Laser Sintering(SLS). Using a laser beam or electron beam to melt a point at a specific location on mental powders or plastic layer. Then bonding into physical layer with a certain thickness layer by layer in the powder bed, and finally forming the solid parts.

Stereo Lithography Apparatus(SLA). In accordance with the information on the section of the three-dimensional digital model of the part, using ultraviolet ray or laser which has particular wavelength and intensity to scan the surface of materials. Then the polymerization reaction took place at the point where can be illuminated. Repeating the process until the objects formed[3].

Electron Beam Melting(EBM). In vacuum environment, scanning and melting the metal powder which is spread over the powder bed by electron beams that are controlled by magnetic deflection coil. Making the materials fuse and solidify in the molten pool, until forming metal parts. 
Selective Laser Melting (SLM). The principle of selective laser melting is similar with the electron beam melting. The difference is that laser beams are used as the light source, not electron beams.

Laser Direct Melting Deposition (LDMD). Taking laser beams as the light source, according to the movement of laser dot, sending metal power into the molten pool result from laser irradiation on metal surface, melting and solidifying to add layer upon layer until the object is fully formed[4].

Three dimensional printing (3D P). Spraying binder on gypsum powder through the ink-jet print head, based on the section contour date. Then spreading a layer of gypsum powder. Repeating the process until the entire profile of the product adhered.

The above technologies are researched and developed by different companies. They differ from each other mainly in raw materials and molding methods[5], as shown in table 1.

Table 1. The classification of 3D printing technology

\begin{tabular}{|c|c|c|}
\hline Types & $\begin{array}{l}\text { Technology } \\
\text { categories }\end{array}$ & Raw materials \\
\hline \multirow[t]{2}{*}{ Extrusion forming } & FDM & $\begin{array}{l}\text { Thermoplastic plastics, eutectic } \\
\text { metal, edible material }\end{array}$ \\
\hline & SLS & $\begin{array}{l}\text { Thermoplastic plastics,ceramic } \\
\text { powder, metal powder }\end{array}$ \\
\hline \multirow[t]{4}{*}{$\begin{array}{l}\text { Forming of } \\
\text { granular materials }\end{array}$} & SLM & $\begin{array}{l}\text { Titanium alloy, cobalt- } \\
\text { chromium alloy, stainless steel, } \\
\text { aluminum }\end{array}$ \\
\hline & EBM & Titanium alloy \\
\hline & $3 \mathrm{D} \mathrm{P}$ & Oulopholite \\
\hline & LDMD & Metal powder \\
\hline Lamination & SLA & Plastic film, metal foil, paper \\
\hline Photopolymerization & LOM & Photosensitive polymer \\
\hline
\end{tabular}

\section{The Applications of 3D Printing Technology}

3D printing technology can be applied to any fields that need models. At present, 3D printing technology has been widely applied in mold manufacturing, aerospace, medical treatment, daily necessities and many other fields.

(1) Mold manufacturing. First, 3D printing is used for making molds directly .Second, parts which are produced by $3 \mathrm{D}$ printing are copied constantly, and generating the desired mold ultimately.

(2) Aerospace science and technology. The applications of 3D printing technology in Aeronautic and space fields can be epitomized as follows. First, producing the specially designed parts and components, with high accuracy and complex structure. Second, 3D printing technology not only can optimize the structures of components on the premise of performance guarantee, but also realize integral manufacture to reduce the weight. Third, it can renovate damaged part rapidly. Furthermore, the components can be renovated more easily, and matching finely.

(3) Medical treatment. 3D printing technology has many applications in the field of medical treatment, mainly includes: medical models, artificial bones, biological organs, teeth, plastic beauty, etc[6]. According to the actual condition of patients, doctors can provide personalized services to satisfy their characteristic requests. For example, medical models which are fabricated by $3 \mathrm{D}$ printing technology can be used for performing simulated surgery. In this way, doctors enable to develop the best surgical program and improve the success rate of operation. 
(4) The field of daily necessities. Cups, tables, chairs, toys, lamps, knives and forks, clothes, shoes, jewelry and other articles for daily use, can be designed personalized to meet the customers' needs and preference. Then using 3D printers to print them out. In addition, 3D printers can be used for printing food. The printed meat is not inferior to the real meat[7].

\section{Advantages of 3D Printing Technology and Its Existing Problems}

\section{Advantages of 3D Printing Technology}

(1) Manufacturing products and components with complex shape and structure. The biggest advantage of 3D printing technology is that designers can fully exert their imagination and creativity which are limited by traditional manufacturing processes.

(2) It is suitable for processing various kinds of products with small batches. In the traditional process of manufacturing products and components, multiple device are used for completing several processing operations. However, using 3D printing technology to produce different kinds of parts, only needs to change the corresponding three-dimensional model and raw materials. The technology has unexampled predominance comparing with the traditional manufacturing technology about the aspect of producing various kinds of products with small batches.

(3) Integrated production and high utilization of materials. Components with integral taking shape can be produced by 3D printing technology[8]. And the entire production process do not need milling, drilling, grinding, cutting and other machining methods. The technology has advantages of reducing the number of components which constitute the product, improving the material utilization rate, shortening the production cycle and reducing the costs of procurement and transportation.

(4) Designing and manufacturing personalized products and service. In order to pursue the lowest costs of productions, the existing methods of mass production produce a large number of standardized products by means of refining division of labor and standardizing operation flow. However, this mode of production is difficult to meet the customs' needs of diversification and personalization. The 3D printing technology makes it possible to design and manufacture personalized products according to personal preferences of customers and different machining requirements of company.

\section{The existing problems of 3D printing technology}

(1) Low precision and low efficiency. The printed products have problems of low precision, low efficiency and poor surface quality. So the technology can not satisfy the requirement of largescale production. In addition, there is a serious conflict between speed and precision of 3D printers [9]. The higher the product precision is, the thinner the thickness of each layer, and printing the same product needs more layers. Time for printing is extended several-fold, and the speed decrease.

(2) The limitation of materials. At present, materials can be used for printing have only a limited diversity. Moreover, the strength, stability, safety, practicality and other performance indicators of the existing materials are not ideal, can not meet the needs well. In addition, there exist certain security problems in the use of existing materials.

(3) Higher costs. The price of 3D printers is comparatively expensive. And most of raw materials and core device need to be imported.

(4) Intellectual property right. Owing to the design process is accomplished by computer, the design achievements can be moved and copied easily. Violating copyright, trademark rights, digitalrights and other rights and interests is easily to take place. Furthermore, the existing intellectual property mechanisms are difficult to meet the future development needs of manufacturing industry. 
(5) Environment protection and energy conservation. Some materials used by 3D printers have been assessed as ultrafine particles, which can get inside lungs and into bloodstream, and will threaten people' $s$ health if they reaches high concentrations. In addition, electricity is the main energy supply of 3D printers. Under the current technical conditions, in the process of producing parts with same mass, the power consumption of 3D printers is about 10 times than using traditional craft methods. If the 3D printing technology is to be applied on a large scale, environmental pollution and energy saving are the problems that need to be considered.

\section{The Development Trend of 3D Printing Technology}

3D printing technology has great potential for development. However, to achieve mass production, the current production efficiency need to be raised by $10 \sim 15$ times at least. And it is hard to implement in a short time. In order to satisfy the market demand, the 3D printing technology should develop towards the following several directions.

(1) 3D printers are developing towards large-scale and miniaturization. On the one hand, 3D printers are becoming large-scale. Giant printers can meet the needs of large scale manufacturing plants, such as aerospace and automobile enterprises[10]. On the other hand, $3 \mathrm{D}$ printers are being developed for miniaturization. The size is smaller, the cost is lower and the operation is more simple.

(2) The diversification development of materials. It is important to improve the performance of 3D printing materials now available. At the same time, developing new materials, such as various composite materials, alloy materials, gradient materials, functional materials, nanophase materials and heterogeneous materials is essential. And trying to make all the materials can be used for 3D printing.

(3) Networking and intelligent. The technology is developing towards networking and intelligent direction. Large-scale distributed network , composed of many small-scale manufacturing enterprises, is good for integrating resources and improving production efficiency. Meanwhile, $3 \mathrm{D}$ printing is also developing to the direction of intelligence. 3D printing software can make different responses according to changes in materials, structures and manufacturing environment.

(4) Using new and renewable energy sources to supply power. At present, most of the processing equipment used in manufacturing industry are driven by electricity. With the rapid development of global economy, environment is deteriorating and natural resources becoming depleted. People have realized that new and renewable energy must be used to provide the necessary impetus to production. In order to realize green and low carbon manufacturing, 3D printers can be powered by solar energy, wind energy, nuclear energy and other new energy sources.

\section{Conclusions}

The development of 3D printing technology means both opportunities and challenges. It may bring revolutionary changes in manufacturing industry in the near future. Turning factory production to social production, converting the production styles from large scale mass production to individual customization production. However, at present, the attractive applications of 3D printing technology are products used for demonstrating. But it is undeniable that 3D printing technology will be an effective complement to current large-scale, standardized industrial system. 


\section{References}

[1] Dongman Yu, Xiaojing Li , Di Wang: submitted to Machinery Design and Manufacture (2011), p. $65-67$

[2] Ling Li, Guangchun Wang: submitted to ShanDong Agricultural Machinery (2005), p. 17-19

[3] Huiling Yuan, Tianrui Zhou: submitted to Southern Metals (2009), p. 24-27

[4] Xuejun Zhang, Siy Tangi, Hengyue Zhao, et al: submitted to Journal of Materials Engineering (2016), p.122-128

[5] Hong Jiang: submitted to Advanced Materials Industry (2013), p. 30-35

[6] Alok Sutradhar, Jaejong Park, Diana Carrau: submitted to Computers in Biology and Medicine (2014), p.8-17

[7] Bo Wang: submitted to Mechanical and Electrical Technology In Chinese (2014), p. 158-160

[8] Xin Li: submitted to Rock Drilling Machinery and Pneumatic Tools In Chinese(2014), p. 31-41

[9] Xueying: Wang, submitted to China High Technology Enterprises In Chinese(2012), p. 3-5

[10] Riyang Guo: submitted to Process Automation Instrumentation In Chinese(2015), p. 5-8 